\title{
Kann auf Zwangsmedikation in der klinischen Praxis verzichtet werden? - Pro
}

\author{
Could Psychiatry Dispense with Involuntary Medication? - Pro
}

\section{Pro}

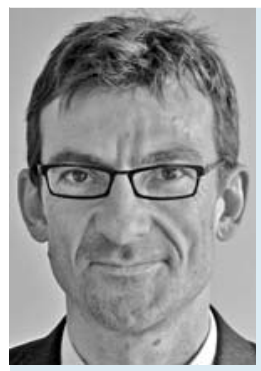

Martin Zinkler

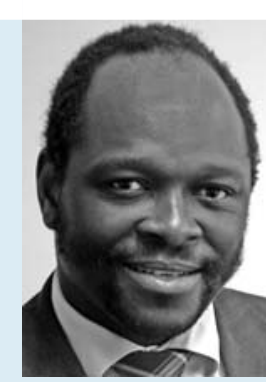

Jose-M. Koussemou

John Conolly brauchte vor 177 Jahren 6 Monate, um im Hanwell Asylum bei London alle mechanischen Zwangsmaßnahmen abzuschaffen - ohne den zwangsweisen Einsatz von Psychopharmaka. Conolly setzte auf Kräftigung, Bewegung und Beschäftigung; dagegen misstraute er den Anhängern von medikamentösen Behandlungen: „Alles, was die Kräfte des Kranken herabsetzt, wirkt ungünstig auf die Krankheit [...] Bewegung im Freien ist nicht hoch genug einzuschätzen; gleiches gilt von der Beschäftigung. Auch scheint es im Allgemeinen, dass die Vorzüge einer langen Reihe auserlesener Pharmakopöe-Artikel mit dem größten Zutrauen nur von solchen wiederholt hervorgehoben werden, die am wenigsten Gelegenheit hatten, sie in großen Instituten zu erproben“" [1].

Nun soll Zwangsmedikation in unseren sehr viel moderneren und personell viel besser ausgestatteten Kliniken unverzichtbar sein? Befürworter der Zwangsmedikation argumentieren oft mit körpermedizinischen Beispielen. Dennoch soll hier zunächst die Zwangsmedikation mit Psychopharmka behandelt werden. Denn sowohl die Verfassungsgerichtsentscheidungen von 2011 (2 BvR 882/09, R\&P 2011, 168 und 2 BvR 633/11, R\&P 2012, 31) als auch die Bundesgerichtshofentscheidungen von 2012 (XII ZB 99/12 und XII ZB 130/12, R\&P 2012, 206) ergingen über Fälle von Zwangsbehandlung mit Neuroleptika bei psychischen Störungen.

\section{Neuroleptika}

Unverzichtbar wäre eine neuroleptische Zwangsbehandlung, wenn sie bei der Behandlung von Psychosen lebensnotwendig wäre. Den Autoren sind jedoch in 25 Jahren allgemein- und akutpsychiatrischer Tätigkeit mit Tausenden von Behandlungen keine solchen Fälle begegnet. Bei katatonen Zuständen kommen weniger gefährliche Behandlungen (z.B. mit Lorazepam) infrage.

Unsere Klinik hat in den 5 Jahren seit den Beschlüssen des Bundesverfassungsgerichts keine neuroleptische Zwangsmedikation mehr durchgeführt. Das gelang mit mehr Geduld, mehr Bereitschaft zur Begleitung durch die Psychose und mehr Toleranz für Widersprüche und Kontroversen mit den Patienten, manchmal mit den Angehörigen und in den Behandlungsteams. Dabei kam es nicht zur häufigeren Anwendung von anderen Zwangsmaßnahmen, wie z.B. Fixierungen. Meistens einigten wir uns allmählich, auf dem Verhandlungsweg über eine niedrigdosierte Behandlung, etwa den Beginn mit einer Dosis von $50 \mathrm{mg}$ Amisulprid - erst einmal für 3 Tage, um zu sehen, wie es vertragen wird.

Wie ist es mit der Behandlung eines Delirs? Delire sind lebensgefährliche Erkrankungen. Wir kennen niemanden, der einmal geäußert hätte, dass er an einem Delir lieber sterben möchte, als sich behandeln zu lassen. Praktisch hat sich die Frage einer Zwangsbehandlung eines Patienten im Delir in den letzten 5 Jahren für uns nicht gestellt, zum einen weil Patienten mit leichteren Delirverläufen ohne weiteres mit neuroleptischer Behandlung einverstanden waren, zum anderen weil schwere lebensgefährliche Delire ohnehin intensivmedizinisch und dann dem mutmaßlichen Willen des Betroffenen folgend behandelt werden.

Die Behandlung mit Neuroleptika greift in seelische Vorgänge ein (Denken, Wollen, Fühlen, Wahrnehmen, Bewegen und Handeln), hat erhebliche Nebenwirkungen, verkürzt bei längerer Dauer wahrschein- lich die Lebenserwartung und scheint den Langzeitverlauf von Psychosen nicht günstig zu beeinflussen [2, 3]. Sie sollte also besonders sorgfältig abgewogen werden und kommt nach unserer Auffassung nur mit einvernehmlicher Entscheidung infrage.

\section{Benzodiazepine}

Ein weiterer Einsatz von Neuroleptika besteht in der Behandlung von akuten Erregungszuständen. Dafür gibt es jedoch mit der parenteralen Gabe von Benzodiazepinen eine wirksame und sichere Alternative.

Ob auf die zwangsweise Gabe von Benzodiazepinen in akuten Erregungszuständen verzichtet werden kann? Wahrscheinlich schon, denn bei Patienten mit substanzbedingten Erregungszuständen wissen sich Psychiater Nacht für Nacht in den Notaufnahmen ihrer Krankenhäuser mehr oder weniger gut zu helfen: je unübersichtlicher die Intoxikation unseres Patienten ist, desto vorsichtiger sind wir mit der Gabe von Benzodiazepinen.

Dennoch halten wir die zwangsweise Gabe von Benzodiazepinen für viel weniger problematisch als die von Neuroleptika, denn sie erfolgt, wenn überhaupt, kurzfristig, ist weniger eingreifend und weniger gefährlich. Wenn doch einmal überdosiert wurde, so gibt es ein wirksames Antidot.

\section{Körperliche Erkrankungen}

Gerne wird bei der Diskussion der Zwangsmedikation unter Psychiatern auf die Behandlung von körperlichen Erkrankungen verwiesen. Nun liegt es uns aber fern, unseren Kollegen der Inneren Medizin oder der Intensivmedizin zu sagen, ob sie auf Zwangsmedikation verzichten können. Denn die medizinischen, rechtlichen und ethischen Fragen bei der Behandlung von lebensbedrohlichen Erkrankungen betreffen alle Ärzte. Ob auf eine Zwangsbehandlung mit Insulin, Anti- 
biotika oder Kortison verzichtet werden kann, ist keine psychiatrische Frage.

Anders als das deutsche Betreuungsrecht sieht die UN-Konvention über die Rechte von Menschen mit Behinderungen [4] keine Einschränkung der Einwilligungsfähigkeit und keine stellvertretende Entscheidung durch eine dritte Person (z.B. einen rechtlichen Betreuer) vor. Ob eine medizinische Behandlung gegen den Willen einer Person überhaupt noch stattfinden kann, ist damit infrage gestellt. Denkbar halten wir, dass im Sinne des Allgemeinen Kommentars zu Artikel 12 [5] eine Behandlung ohne den aktuellen Willen der Person zustandekommen kann, wenn die bestmögliche Interpretation des früher bekundeten Willens und der Einstellungen (preferences) der Person für eine Behandlung spricht.

Rasch sind gerichtliche genehmigte Zwangsbehandlungen in der deutschen Psychiatrie mit der Neufassung des $§ 1906$ BGB wieder aufgenommen werden, in Bayern mit Häufigkeit von etwa 0,5\% aller Beandlungsfälle [6]. Unsere Erfahrung ist jedoch, dass neuroleptische Zwangsbehandlung zu Drehtüreffekten führt, während ein Verzicht darauf die Möglichkeit eröffnet, sich geduldig aufeinander einzulassen, neue Kommunikationstechniken zu lernen und über Verständnis, Optimismus und Kreativität neue Wege in der Be- handlung von schwer kranken Menschen zu gehen [7].

Seit 20 Jahren treffen sich Psychiaterinnen, Psychologen, Sozialarbeiterinnen, Krankenpfleger, Psychiatrieerfahrene und Angehörige bei der Tagung „Die subjektive Seite der Schizophrenie“. Wer daran teilnimmt spürt, wie groß das Interesse an einer Weiterentwicklung der Behandlung ist. Dennoch fristen viele Ansätze (offener Dialog, Soteria, Peer-Support, Unterstützung beim Absetzen von Neuroleptika) ein Nischendasein, während die Mainstream-Psychiatrie im Ernstfall auf Zwangsmaßnahmen zurückgreift. Das führt dann dazu, dass diejenigen sich rechtfertigen müssen, die auf Zwang, Einsperren und Zwangsbehandlung verzichten. Es ist höchste Zeit, dass sich diese Verhältnisse umkehren.

\section{Literatur}

1 Conolly J. Behandlung der Irren ohne mechanischen Zwang. Lahr: Verlag von M. Schauenburg \& CS; 1860: 39

2 Whitaker $R$. The case against antipsychotic drugs: a 50-year record of doing more harm than good. Medical Hypotheses 2004; 62: $5-13$

3 Wunderink L, Nieboer RM, Wiersma D et al. Recovery in Remitted First-Episode Psychosis at 7 Years of Follow-up of an Early Dose Reduction/Discontinuation or Maintenance Treatment Strategy. Long-term Follow-up of a 2-Year Randomized Clinical Trial. JAMA Psychiatry10.1001/jamapsychiatry.2013.19
4 Übereinkommen über die Rechte von Menschen mit Behinderungen vom 13. Dezember 2006. Bundesgesetzblatt (BGBL); 2008: II: 1419

5 United Nations, Committee on the Rights of Persons with Disabilities. General comment No. 1 (2014) Article 12: Equal recognition before the law. 2014: CRPD/C/GC/1

6 Albus M, Brieger P, Schreiber W. Zwangsbehandlung mit Psychopharmaka. Auswirkungen der Gesetzesänderung zum 18.02.2013 auf psychiatrische Abteilungen und Kliniken mit Vollversorgungsauftrag in Bayern. Recht \& Psychiatrie 2015; 33: 193-197

7 Zinkler M, Koussemou JM. Nach den Entscheidungen des Bundesverfassungsgerichts und des Bundesgerichtshofs zur Zwangsbehandlung - drei Fallberichte. Recht \& Psychiatrie 2013; 31: 76 - 79

Sie haben eine eigene Meinung zu diesem Thema? Dann schreiben Sie uns an: psychiat-praxis@thieme.de!

\section{Korrespondenzadresse}

\section{Dr. med. Martin Zinkler}

Kliniken Landkreis Heidenheim gGmbH Schlosshaustraße 100

89522 Heidenheim

martin.zinkler@kliniken-heidenheim.de

Bibliografie

DoI http://dx.doi.org/

10.1055/s-0042-105549

Psychiat Prax 2016; 43: 187-188

(c) Georg Thieme Verlag KG

Stuttgart · New York

ISSN 0303-4259 